
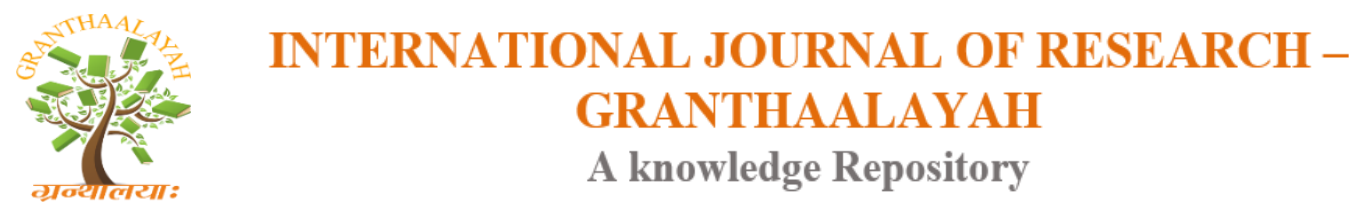

Science

\title{
A CLASS OF TWO-SAMPLE SCALE TESTS BASED ON U-STATISTICS
}

\author{
Sharada V. Bhat ${ }^{* 1}$, Shashank D. Shindhe ${ }^{2}$ \\ ${ }^{* 1,2}$ Department of Statistics, Karnatak University, Dharwad, Karnataka, India
}

\begin{abstract}
A class of tests based on U-statistic is proposed for two-sample scale problem. The U-statistic is function of extremes of subsamples taken from random samples of two absolutely continuous distributions. The asymptotic distribution, null distribution and efficacy of the class of tests are studied. A comparison of its performance with respect to other tests is studied in terms of Pitman ARE and small sample performance through its empirical power. Application of the class of tests is illustrated through an example.
\end{abstract}

Keywords: Empirical Power; Pitman ARE; Scale Problem; Two-Sample; U-Statistics.

Cite This Article: Sharada V. Bhat, and Shashank D. Shindhe. (2019). "A CLASS OF TWOSAMPLE SCALE TESTS BASED ON U-STATISTICS." International Journal of Research - Granthaalayah, 7(9), 138-147. https://doi.org/10.29121/granthaalayah.v7.i9.2019.569.

\section{Introduction}

Variability is a fundamental component of almost all the phenomena observed in Nature. In some cases it is the outcome to be studied while in some other cases it is pre-existent. For instance, in a clinical trial studying variances in the effects of a treatment when subjects are provided with different dosages of a single drug would be an attempt to study the variability in the outcomes. On the other hand, studying variances in the effects of the same drug when subjects are provided with same dosages would be studying individual differences among the subjects. In the first case variance is induced while in the second, variance is a pre-existent nuisance. Whatever the case be, testing for variances among two populations is a fundamental problem in statistical inference.

Suppose, $X_{1}, X_{2}, \ldots, X_{m_{1}}$ and $Y_{1}, Y_{2}, \ldots, Y_{m_{2}}$ are two independent random samples from two populations with absolutely continuous distribution functions $F(x)$ and $G(x)$ such that $G(x)=$ $F\left(\frac{x}{\sigma}\right), \sigma>0$. Then testing for possible differences among the two populations with respect to (wrt) $\sigma$ by defining $H_{0}: \sigma=1$ against $H_{1}: \sigma>1$ constitute two-sample scale problem.

Under parametric setup, Snedecor's F-test (given in Rohatgi and Saleh (2001)) is used to test $H_{0}$ against $H_{1}$. Tests due to Mood (1954) and Siegel and Tukey (1960) are some earlier nonparametric tests based on ranks. Many distribution-free tests based on U-statistics for testing $H_{0}$ against $H_{1}$ are 
available in the literature. Some of them are due to, Sukhatme (1957, 1958), Deshpande and Kusum (1984). Kusum (1985), Kochar and Gupta (1986), Mehra and Rao (1992), Shetty and Bhat (1993), Shetty and Pandit (2004), Mahajan et. al. (2011), Kossler and Narinder Kumar (2016), Narinder Kumar and Goyal (2018), Bhat et. al. (2018) and Bhat and Shindhe (2019). Lehmann (1951) established asymptotic distribution of the two-sample U-statistics. An extensive review of tests for two-sample scale problem is given by Duran (1976).

In this paper, we propose a class of distribution-free tests, $A\left(k_{1}, k_{2}\right)$ based on U-statistics which is a function of extremes of subsamples of sizes $k_{1}$ and $k_{2}$ respectively from $X$ and $Y$ samples. The class of tests with its alternative form is given in section 2. Section 3 contains the distributional properties of $A\left(k_{1}, k_{2}\right)$, section 4 deals with its efficacy, Pitman ARE and empirical power. In sections 5, we furnish conclusions along with an illustration of the application of $A\left(k_{1}, k_{2}\right)$.

\section{A Class of Distribution-Free Tests}

In this section, we propose a class of tests based on two-sample U-statistic being a function of subsample extremes since the information contained in the tails of the distribution plays an important role in detecting difference in scales. The proposed class of tests is given by

$A\left(k_{1}, k_{2}\right)=\left(\left(\begin{array}{c}m_{1} \\ k_{1}\end{array}\right)\left(\begin{array}{c}m_{2} \\ k_{2}\end{array}\right)\right)^{-1} \sum_{\mathcal{D}} \phi\left(X_{i_{1}}, X_{i_{2}}, \ldots, X_{i_{k_{1}}} ; Y_{j_{1}}, Y_{j_{2}}, \ldots, Y_{j_{k_{2}}}\right)$

where, $\mathcal{D}$ denotes summation over all possible $\left(\begin{array}{c}m_{1} \\ k_{1}\end{array}\right)\left(\begin{array}{c}m_{2} \\ k_{2}\end{array}\right)$ combinations of $X_{1}, X_{2}, \ldots, X_{k_{1}} \quad$ and $Y_{1}, Y_{2}, \ldots, Y_{k_{2}}$ observations,

$\phi\left(x_{1}, x_{2}, \ldots, x_{k_{1}} ; y_{1}, y_{2}, \ldots, y_{k_{2}}\right)=\left\{\begin{array}{rr}1 \quad \text { if } 0<x_{\left(k_{1}\right)}^{+}<y_{\left(k_{2}\right)}^{+}, x_{i}, y_{j}>0 \\ \\ \text { or } y_{(1)}^{-}<x_{(1)}^{-}<0, x_{i}, y_{j}<0 \\ -1 \quad \begin{array}{l}\text { if } 0<x_{(1)}^{+}<y_{(1)}^{+}, x_{i}, y_{j}>0 \\ \text { or } y_{\left(k_{2}\right)}^{-}<x_{\left(k_{1}\right)}^{-}<0, x_{i}, y_{j}<0 \\ \text { Otherwise }\end{array}\end{array}\right.$

$i=1,2, \ldots, k_{1}, j=1,2, \ldots, k_{2}, x_{\left(k_{1}\right)}^{+}$and $\bar{x}_{\left(k_{1}\right)}^{-}\left(y_{\left(k_{2}\right)}^{+}\right.$and $\left.\bar{y}_{\left(k_{2}\right)}^{-}\right)$respectively denote the maximum order statistics of positive and negative $X(Y)$ observations, $x_{(1)}^{+}$and $x_{(1)}^{-}\left(y_{(1)}^{+}\right.$and $\left.y_{(1)}^{-}\right)$ respectively denote minimum order statistics of positive and negative $X(Y)$ observations and $k_{1}\left(k_{2}\right)$ is the size of the subsample from $X(Y)$ sample satisfying $\quad 1 \leq k_{1} \leq m_{1}(1 \leq$ $\left.k_{2} \leq m_{2}\right)$.

The newly proposed class of tests $A\left(k_{1}, k_{2}\right)$ can also be obtained from tests $B_{h}\left(k_{1}, k_{2}\right)$ and $B_{l}\left(k_{1}, k_{2}\right)$ proposed in Bhat and Shindhe (2019). It can be expressed as

$A\left(k_{1}, k_{2}\right)=B_{h}\left(k_{1}, k_{2}\right)-B_{l}\left(k_{1}, k_{2}\right)$

where

$B_{h}\left(k_{1}, k_{2}\right)=\left(\left(\begin{array}{c}m \\ k_{1}\end{array}\right)\left(\begin{array}{c}n \\ k_{2}\end{array}\right)\right)^{-1} \sum_{\mathcal{D}} \varphi_{h}\left(X_{i_{1}}, X_{i_{2}}, \ldots, X_{i_{k_{1}}} ; Y_{j_{1}}, Y_{j_{2}}, \ldots, Y_{j_{k_{2}}}\right)$, 
$B_{l}\left(k_{1}, k_{2}\right)=\left(\left(\begin{array}{c}m \\ k_{1}\end{array}\right)\left(\begin{array}{c}n \\ k_{2}\end{array}\right)\right)^{-1} \sum_{\mathcal{D}} \varphi_{l}\left(X_{i_{1}}, X_{i_{2}}, \ldots, X_{i_{k_{1}}} ; Y_{j_{1}}, Y_{j_{2}}, \ldots, Y_{j_{k_{2}}}\right)$,

$\varphi_{h}\left(x_{1}, x_{2}, \ldots, x_{k_{1}} ; y_{1}, y_{2}, \ldots, y_{k_{2}}\right)=\left\{\begin{array}{rrr}1 & \text { if } 0<x_{\left(k_{1}\right)}^{+}<y_{\left(k_{2}\right)}^{+}, \quad x_{i}, y_{j}>0 \\ -1 & \text { if } y_{\left(k_{2}\right)}^{-}<x_{\left(k_{1}\right)}^{-}<0, & x_{i}, y_{j}<0 \\ 0 & \text { Otherwise }\end{array}\right.$

and $\varphi_{l}\left(x_{1}, x_{2}, \ldots, x_{k_{1}} ; y_{1}, y_{2}, \ldots, y_{k_{2}}\right)=\left\{\begin{array}{ccc}1 & \text { if } 0<x_{(1)}^{+}<y_{(1)}^{+}, \quad x_{i}, y_{j}>0 \\ -1 & \text { if } y_{(1)}^{-}<x_{(1)}^{-}<0, & x_{i}, y_{j}<0, \\ 0 & \text { Otherwise }\end{array}\right.$

The class of tests $A\left(k_{1}, k_{2}\right)$ is distribution-free for $1 \leq k_{1} \leq m_{1}$ and $1 \leq k_{2} \leq m_{2}$ and large values of the test statistic are significant for testing $H_{0}$ against $H_{1}$.

Assuming that there are no ties, following Bhat (1995) an alternative form of $A^{*}\left(k_{1}, k_{2}\right)=$ $\left(\begin{array}{c}m_{1} \\ k_{1}\end{array}\right)\left(\begin{array}{c}m_{2} \\ k_{2}\end{array}\right) A\left(k_{1}, k_{2}\right)$ based on ordered ranks is given by

$A^{*}\left(k_{1}, k_{2}\right)=A_{1}^{*}-A_{2}^{*}$

Where,

$A_{1}^{*}=\sum_{i_{1}=1}^{m_{1}^{+}} \sum_{j_{1}=0}^{k_{2}-1}\left(\begin{array}{c}i_{1}-1 \\ k_{1}-1\end{array}\right)\left(\begin{array}{c}R_{\left(i_{1}\right)}^{+}-i_{1} \\ k_{2}-j_{1}-1\end{array}\right)\left(\begin{array}{c}m_{2}^{+}-R_{\left(i_{1}\right)}^{+}+i_{1} \\ j_{1}+1\end{array}\right)+\sum_{i_{2}=1}^{m_{2}^{-}}\left(\begin{array}{c}m_{2}^{-}-i_{2} \\ k_{2}-1\end{array}\right)\left(\begin{array}{c}m_{1}^{-}-S_{\left(i_{2}\right)}^{-}+i_{2} \\ k_{1}\end{array}\right)$,

$A_{2}^{*}=\sum_{i_{1}=1}^{m_{1}^{+}}\left(\begin{array}{c}m_{1}^{+}-i_{1} \\ k_{1}-1\end{array}\right)\left(\begin{array}{c}m_{2}^{+}-R_{\left(i_{1}\right)}^{+}+i_{1} \\ k_{2}\end{array}\right)+\sum_{i_{2}=1}^{m_{2}^{-}} \sum_{j_{2}=0}^{k_{1}-1}\left(\begin{array}{c}i_{2}-1 \\ k_{2}-1\end{array}\right)\left(\begin{array}{c}S_{\left(i_{2}\right)}^{-}-i_{2} \\ k_{1}-j_{2}-1\end{array}\right)\left(\begin{array}{c}m_{1}^{-}-S_{\left(i_{2}\right)}^{-}+i_{2} \\ j_{2}+1\end{array}\right)$,

$m_{1}=m_{1}^{+}+m_{1}^{-}, m_{2}=m_{2}^{+}+m_{2}^{-}, \quad R_{\left(i_{1}\right)}^{+}$is the rank of $X_{\left(i_{1}\right)}^{+}$in the joint rankings of $X_{(1)}^{+}, X_{(2)}^{+}, \ldots, X_{\left(m_{1}^{+}\right)}^{+}, Y_{(1)}^{+}, \ldots, Y_{\left(m_{2}^{+}\right)}^{+}$such that $X_{(1)}^{+}<X_{(2)}^{+}<\cdots<X_{\left(m_{1}^{+}\right)}^{+}$and $Y_{(1)}^{+}<Y_{(2)}^{+} \ldots<Y_{\left(m_{2}^{+}\right)}^{+}$ respectively are ordered positive $X$ and $Y$ observations and $S_{\left(i_{2}\right)}^{-}$is the rank of $Y_{\left(i_{2}\right)}^{-}$in the joint rankings of $X_{(1)}^{-}, X_{(2)}^{-}, \ldots, X_{\left(m_{1}^{-}\right)}^{-}, Y_{(1)}^{-}, \ldots, Y_{\left(m_{2}^{-}\right)}^{-}$such that $X_{(1)}^{-}<X_{(2)}^{-}<\cdots<X_{\left(m_{1}^{-}\right)}^{-}$and $Y_{(1)}^{-}<$ $Y_{(2)}^{-}<\cdots<Y_{\left(m_{2}^{-}\right)}^{-}$respectively are ordered negative $X$ and $Y$ observations.

\section{Distribution of $A\left(k_{1}, k_{2}\right)$}

In this section, we derive the null mean and asymptotic distribution of the proposed class of tests. Also, we obtain the null distribution of $A^{*}\left(k_{1}, k_{2}\right)$ using Monte-Carlo simulation.

The mean of $A\left(k_{1}, k_{2}\right)$ is given by,

$E\left[A\left(k_{1}, k_{2}\right)\right]=P\left(0<X_{\left(k_{1}\right)}<Y_{\left(k_{2}\right)}\right)+P\left(Y_{(1)}<X_{(1)}<0\right)-P\left(0<X_{(1)}<Y_{(1)}\right)-P\left(Y_{\left(k_{2}\right)}<\right.$ $\left.X_{\left(k_{1}\right)}<0\right)$ 
and the null mean is given by

$E_{H_{0}}\left[A\left(k_{1}, k_{2}\right)\right]=\mu_{0}=\frac{2\left(k_{2}-k_{1}\right)}{k_{1}+k_{2}}$.

According to Lehmann (1951) as $N \rightarrow \infty$ such that $0<\lambda=\lim _{N \rightarrow \infty} \frac{m}{N}<1, \sqrt{N}\left[A\left(k_{1}, k_{2}\right)-\mu_{0}\right]$ follows asymptotic normal distribution with mean zero and variance

$\sigma^{2}=\frac{k_{1}^{2} \zeta_{10}}{\lambda}+\frac{k_{2}^{2} \zeta_{01}}{1-\lambda}$

Where,

$\zeta_{10}=\operatorname{Cov}\left[\phi\left(X_{1}, \ldots, X_{k_{1}} ; Y_{1}, \ldots, Y_{k_{2}}\right), \phi\left(X_{1}, X_{k_{1}+1}, \ldots, X_{2 k_{1}-1} ; Y_{k_{2}+1}, \ldots, Y_{2 k_{2}}\right)\right]$

and $\zeta_{01}=\operatorname{Cov}\left[\phi\left(X_{1}, \ldots, X_{k_{1}} ; Y_{1}, \ldots, Y_{k_{2}}\right), \phi\left(X_{k_{1}+1}, \ldots, X_{2 k_{1}} ; Y_{1}, Y_{k_{2}+1}, \ldots, Y_{2 k_{2}-1}\right)\right]$.

Defining,

$\eta_{1}=P\left(0<\operatorname{Maximum}\left(x, X_{2}, \ldots, X_{k_{1}}\right)<\operatorname{Maximum}\left(Y_{1}, \ldots, Y_{k_{2}}\right)\right)$,

$\eta_{2}=P\left(\operatorname{Minimum}\left(Y_{1}, \ldots, Y_{k_{2}}\right)<\operatorname{Minimum}\left(x, X_{2}, \ldots, X_{k_{1}}\right)<0\right)$,

$\eta_{3}=P\left(0<\operatorname{Minimum}\left(x, X_{2}, \ldots, X_{k_{1}}\right)<\operatorname{Minimum}\left(Y_{1}, \ldots, Y_{k_{2}}\right)\right)$,

and $\eta_{4}=P\left(\operatorname{Maximum}\left(Y_{1}, \ldots, Y_{k_{2}}\right)<\operatorname{Maximum}\left(x, X_{2}, \ldots, X_{k_{1}}\right)<0\right)$,

We get

$$
\begin{aligned}
& \zeta_{10}=\int_{-\infty}^{\infty}\left[\left(\eta_{1}+\eta_{2}\right)-\left(\eta_{3}+\eta_{4}\right)\right]^{2} d 2 F(x)-\mu_{0}^{2} \\
& =\frac{2 k_{2}^{2}}{\left(k_{1}+k_{2}-1\right)^{2}}\left[\frac{k_{1}+k_{2}-2}{k_{1}+k_{2}}+\frac{1}{2 k_{1}+2 k_{2}-1}\right] \\
& +\frac{2}{\left(k_{1}+k_{2}-1\right)^{2}}\left[\left(k_{1}-1\right)^{2}+\frac{k_{2}^{2}}{2 k_{1}+2 k_{2}-1}+\frac{2 k_{2}\left(k_{1}-1\right)}{k_{1}+k_{2}}\right]-\frac{2\left(k_{1}-k_{2}\right)^{2}}{\left(k_{1}+k_{2}\right)^{2}} \\
& -\frac{4 k_{1} k_{2}}{\left(k_{1}+k_{2}\right)^{2}} \\
& =\frac{4 k_{2}^{2}}{\left(k_{1}+k_{2}-1\right)^{2}}\left[\frac{1}{2 k_{1}+2 k_{2}-1}-\frac{1}{\left(k_{1}+k_{2}\right)^{2}}\right] \text {. }
\end{aligned}
$$

On similar lines by defining

$\eta_{1}^{\prime}=P\left(0<\operatorname{Maximum}\left(X_{1}, \ldots, X_{k_{1}}\right)<\operatorname{Maximum}\left(y, Y_{2}, \ldots, Y_{k_{2}}\right)\right)$,

$\eta_{2}^{\prime}=P\left(\operatorname{Minimum}\left(y, Y_{2}, \ldots, Y_{k_{2}}\right)<\operatorname{Minimum}\left(X_{1}, \ldots, X_{k_{1}}\right)<0\right)$,

$\eta_{3}^{\prime}=P\left(0<\operatorname{Minimum}\left(X_{1}, \ldots, X_{k_{1}}\right)<\operatorname{Minimum}\left(y, Y_{2}, \ldots, Y_{k_{2}}\right)\right)$,

and $\eta_{4}^{\prime}=P\left(\operatorname{Maximum}\left(y, Y_{2}, \ldots, Y_{k_{2}}\right)<\operatorname{Maximum}\left(X_{1}, \ldots, X_{k_{1}}\right)<0\right)$, 
We get

$$
\begin{aligned}
& \zeta_{01}=\int_{-\infty}^{\infty}\left[\left(\eta_{1}^{\prime}+\eta_{2}^{\prime}\right)-\left(\eta_{3}^{\prime}+\eta_{4}^{\prime}\right)\right]^{2} d 2 F(x)-\mu_{0}^{2} \\
& =\frac{4 k_{1}^{2}}{\left(k_{1}+k_{2}-1\right)^{2}}\left[\frac{1}{2 k_{1}+2 k_{2}-1}-\frac{1}{\left(k_{1}+k_{2}\right)^{2}}\right] .
\end{aligned}
$$

From (7) and (8), we observe that $k_{1}^{2} \zeta_{10}=k_{2}^{2} \zeta_{01}$.

Hence, $\sigma^{2}=\frac{k_{1}^{2} \zeta_{10}}{\lambda(1-\lambda)}$.

For, $k_{1}=k_{2}=k \sigma^{*^{2}}=\frac{k^{2}}{\lambda(1-\lambda)(4 k-1)}$

The null distribution of $A^{*}\left(k_{1}, k_{2}\right)$ is obtained by generating 10000 random samples from uniform distribution for different values of $m_{1}, m_{2}, m_{1}^{+}, m_{2}^{+}, k_{1}$ and $k_{2}$ using (3) and is presented in figure 1 .
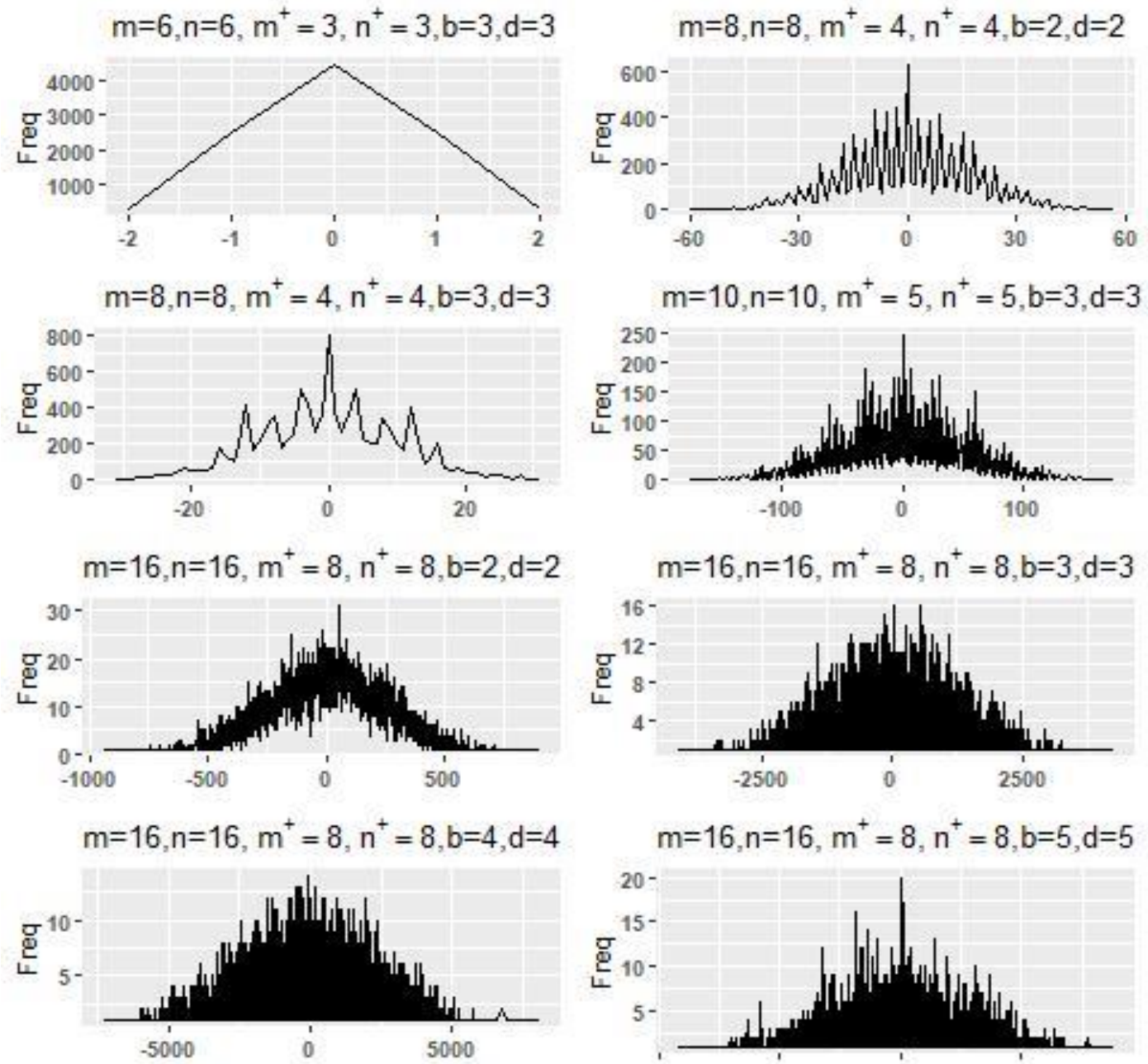

Figure 1: Null distribution of $A^{*}\left(k_{1}, k_{2}\right)$. 
We observe from the figure that the distribution of $A^{*}\left(k_{1}, k_{2}\right)$ is symmetric and is asymptotically normal.

\section{Performance of The Proposed Class Of Tests}

We assess the performance of the proposed class of tests in terms of large and small samples. The large sample performance is assessed using Pitman ARE while the small sample performance in terms of empirical power.

Pitman ARE of $A\left(k_{1}, k_{2}\right)$ wrt any other test $T$ for the sequence of alternatives $F\left(\frac{x}{\sigma_{N}}\right), \sigma_{N}=1+$ $\left(\frac{\sigma}{\sqrt{N}}\right)$, is given by

$A R E\left[A\left(k_{1}, k_{2}\right), T\right]=\frac{e\left[A\left(k_{1}, k_{2}\right)\right]}{e(T)}$,

Where,

$e\left[A\left(k_{1}, k_{2}\right)\right]=\frac{k_{1}^{2} k_{2}^{2}\left[\left(I_{1}-I_{2}\right)-\left(I_{3}-I_{4}\right)\right]^{2}}{\sigma^{2}}$,

$I_{1}=\int_{0}^{\infty} x(2 F(x)-1)^{k_{1}+k_{2}-2}(2 f(x))^{2} d x$,

$I_{2}=\int_{-\infty}^{0} x(1-2 F(x))^{k_{1}+k_{2}-2}(2 f(x))^{2} d x$,

$I_{3}=\int_{0}^{\infty} x(2 \bar{F}(x))^{k_{1}+k_{2}-2}(2 f(x))^{2} d x, \bar{F}(x)=1-F(x)$

and $I_{4}=\int_{-\infty}^{0} x(2 F(x))^{k_{1}+k_{2}-2}(2 f(x))^{2} d x$.

The efficacy values of $A\left(k_{1}, k_{2}\right)$ are presented in table 1 of appendix for $k=k_{1}+k_{2}$ as efficacy values are the same for $k$ for varying values of $k_{1}$ and $k_{2}$. The performance of the proposed class of tests wrt tests due to Mood (M, 1954), Siegel and Tukey (ST, 1960), Deshpande and Kusum $\left(T_{1}, 1984\right)$, Kusum $\left(T_{2}, 1985\right)$, Kochar and Gupta $\left(T_{3}\left(b_{1}, b_{2}\right), 1986\right)$, Shetty and Bhat $\left(T_{4}(3, c)\right.$, 1993) Bhat et. al. $\left(T_{5}\left(d_{1}, d_{2}\right), 2018\right)$ and Bhat and Shindhe $\left(B_{h}\left(k_{1}, k_{2}\right), 2019\right)$ is studied. The values of Pitman ARE are computed and are furnished in tables 2 and 3 in appendix. We take, $b=$ $b_{1}+b_{2}$ and $d=d_{1}+d_{2}$. The empirical power is given in table 4 of appendix.

We observe that, $\operatorname{ARE}\left(A\left(k_{1}, k_{2}\right), B_{h}\left(k_{1}, k_{2}\right)\right)=2$.

It is observed from table 2 that, under uniform distribution $A\left(k_{1}, k_{2}\right)$ outperforms $S T$ and it outperforms $M$ for $k \geq 5$. Under normal distribution it outperforms $M, S T, T_{1}$ and $T_{2}$. The ARE of $A\left(k_{1}, k_{2}\right)$ wrt $M, S T, T_{1}$ and $T_{2}$ are increasing for increasing values of $k$.

Table 3 shows that, $A\left(k_{1}, k_{2}\right)$ is better than $T_{3}\left(b_{1}, b_{2}\right)$ under normal distribution. The ARE is increasing with increasing $k$, but decreases for a given value of $k$ as $b$ increases. It is better than $T_{4}(3, c)$ for uniform and normal distributions. The ARE of $A\left(k_{1}, k_{2}\right)$ wrt $T_{4}(3, c)$ is increasing as $k$ increases, but decreases as $c$ increases for a given value of $k$. 
The ARE of $A\left(k_{1}, k_{2}\right)$ wrt $T_{5}\left(d_{1}, d_{2}\right)$ is increasing with increasing values of $k$ and $d$. The class of tests $A\left(k_{1}, k_{2}\right)$ outperforms $T_{5}\left(d_{1}, d_{2}\right)$ under normal distribution and under uniform distribution when $k \geq 4, d \geq 10$.

As far as small sample performance is concerned, from table 4, we see that the empirical power of $A^{*}\left(k_{1}, k_{2}\right)$ is higher for smaller values of $m_{1}, m_{2}, m_{1}^{+}, m_{2}^{+}, k_{1}, k_{2}$ and it decreases as they increase. It is high for normal, logistic, Laplace and Cauchy distributions as compared to uniform distribution when $\sigma \leq 1.2$ whereas, empirical power under uniform distribution is higher as $\sigma$ increases for larger values of $m, n, m_{1}^{+}$and $m_{2}^{+}$.

\section{Conclusions}

To illustrate the application of the proposed class of tests, following example of two samples of gasket diameters from two brands given in Deshpande et. al. (1995) is considered. The measurements of diameters are recorded as deviations from a common median.

Brand A: $-1.13,-1.31,-1.12,-1.03,-0.5,0.26,0.38,0.64,1.05,1.5,-0.9,0.95$

Brand B: $-0.06,-0.05,-0.11,-0.02,-1.06,-1.02,-1.12,0.04,0.24,0.54,0.89,0.83,0.99,1.05$,

$$
1.12,-0.89
$$

The p-values of different tests are computed using R-program and are given below.

\begin{tabular}{|l|l|l|l|l|l|c|c|}
\hline Test & $A^{*}(2,2)$ & $A^{*}(2,3)$ & $A^{*}(3,3)$ & $A^{*}(3,4)$ & $A^{*}(4,4)$ & $M$ & $F$ \\
\hline p-value & 0.0816 & 0.1039 & 0.0575 & 0.1399 & 0.1701 & 0.1602 & 0.3765 \\
\hline
\end{tabular}

It is observed that, some members of the proposed class of tests have smaller p-values than those of $M$ and $F$-tests. As p-values are smaller for smaller subsample sizes, the choice of the test statistics with smaller subsample sizes is helpful in testing the difference in variability among two samples.

We conclude that,

The proposed class of tests $A\left(k_{1}, k_{2}\right)$ based on U-Statistic as function of subsample extremes is distribution-free and its large values are significant for testing $H_{0}$ against $H_{1}$.

The null distribution of the class of tests is symmetric and follows asymptotic normal distribution. $A\left(k_{1}, k_{2}\right)$ is easily obtained by $B_{h}\left(k_{1}, k_{2}\right), B_{l}\left(k_{1}, k_{2}\right)$ and its asymptotic efficiency is 2 times that of $B_{h}\left(k_{1}, k_{2}\right)$.

The class of tests outperforms $M, S T, T_{4}(3, c), T_{5}\left(d_{1}, d_{2}\right)$ tests under uniform, normal distribution and $T_{1}, T_{2}, T_{3}\left(b_{1}, b_{2}\right)$ tests under normal distribution.

Some members of $A\left(k_{1}, k_{2}\right)$ yield smaller p-values than $M$ and $F$ tests.

The proposed class of tests have higher power for medium tailed distributions when $\sigma \leq 1.2$ whereas, for larger values of $m_{1}, m_{2}, m_{1}^{+}, m_{2}^{+}$the power under light tailed distributions is higher for $\sigma>1.2$. 


\section{Appendices}

Table 1: Efficacies of $A\left(k_{1}, k_{2}\right)$ for different values of $k_{1}, k_{2}$ and various distributions.

\begin{tabular}{|l|l|l|l|l|l|l|}
\hline $\boldsymbol{k}$ & Uniform & Triangular & Exponential & Normal & Logistic & Laplace \\
\hline 4 & 3.1111 & 0.2844 & 0.0123 & 4.3453 & 0.1609 & 0.0864 \\
\hline 5 & 5.0625 & 0.4967 & 0.0210 & 6.6074 & 0.2430 & 0.1314 \\
\hline 6 & 7.0400 & 0.6605 & 0.0270 & 8.4134 & 0.3067 & 0.1673 \\
\hline 7 & 9.0278 & 0.7768 & 0.0307 & 9.8285 & 0.3548 & 0.1955 \\
\hline 8 & 11.0204 & 1.3375 & 0.0510 & 10.9358 & 0.3909 & 0.2175 \\
\hline 9 & 13.0156 & 1.8534 & 0.0682 & 11.8053 & 0.4181 & 0.2347 \\
\hline 10 & 15.0123 & 2.2971 & 0.0817 & 12.4909 & 0.4384 & 0.2484 \\
\hline
\end{tabular}

Table 2: ARE of $A\left(k_{1}, k_{2}\right)$ wrt $M, S T, T_{1}$ and $T_{2}$.

\begin{tabular}{|l|c|c|c|c|c|c|}
\hline \multirow{2}{*}{$\boldsymbol{k}$} & \multicolumn{2}{|l|}{ Uniform } & \multicolumn{2}{l|}{ Normal } \\
\cline { 2 - 7 } & $\boldsymbol{M}$ & $\boldsymbol{S T}$ & $\boldsymbol{M}$ & $\boldsymbol{S} \boldsymbol{T}$ & $\boldsymbol{T}_{\mathbf{1}}$ & $\boldsymbol{T}_{\mathbf{2}}$ \\
\hline 4 & 0.6222 & 1.0371 & 2.8591 & 3.5740 & 3.5767 & 2.5868 \\
\hline 5 & 1.0125 & 1.6876 & 4.3475 & 5.4346 & 5.4386 & 3.9334 \\
\hline 6 & 1.4080 & 2.3468 & 5.5358 & 6.9200 & 6.9252 & 5.0086 \\
\hline 7 & 1.8056 & 3.0095 & 6.4670 & 8.0840 & 8.0900 & 5.8510 \\
\hline 8 & 2.2041 & 3.6737 & 7.1956 & 8.9948 & 9.0014 & 6.5102 \\
\hline 9 & 2.6031 & 4.3388 & 7.7677 & 9.7099 & 9.7171 & 7.0278 \\
\hline 10 & 3.0025 & 5.0044 & 8.2188 & 10.2738 & 10.2814 & 7.4359 \\
\hline
\end{tabular}

Table 3: ARE of $A\left(k_{1}, k_{2}\right)$ wrt $T_{3}\left(b_{1}, b_{2}\right), T_{4}(3, c)$ and $T_{5}\left(d_{1}, d_{2}\right)$.

\begin{tabular}{|c|c|c|c|c|c|c|c|c|}
\hline \multirow[t]{2}{*}{$\boldsymbol{k}$} & \multicolumn{2}{|c|}{$T_{3}\left(b_{1}, b_{2}\right)$} & \multicolumn{3}{|c|}{$T_{4}(3, c)$} & \multicolumn{3}{|c|}{$T_{5}\left(d_{1}, d_{2}\right)$} \\
\hline & $b$ & Normal & $c$ & Uniform & Normal & $d$ & Uniform & Normal \\
\hline \multirow[t]{3}{*}{4} & 5 & 2.7751 & 2 & 1.3333 & 3.6609 & 6 & 0.8822 & 2.0605 \\
\hline & 6 & 2.6758 & 5 & 1.1328 & 3.3955 & 8 & 0.9633 & 2.1375 \\
\hline & 7 & 2.5872 & 7 & 0.9202 & 3.1073 & 10 & 1.0209 & 2.1962 \\
\hline \multirow[t]{3}{*}{5} & 5 & 4.2197 & 2 & 2.1696 & 3.6609 & 6 & 1.4355 & 331 \\
\hline & 6 & 4.0688 & 5 & 1.8433 & 3.3955 & 8 & 1.5675 & 3.2502 \\
\hline & 7 & 3.9341 & 7 & 1.4973 & 3.1073 & 10 & 1.6612 & 3.3395 \\
\hline \multirow[t]{3}{*}{6} & 5 & 5.3730 & 2 & 3.0171 & 7.0881 & 6 & 1.9962 & 3.9895 \\
\hline & 6 & 5.1809 & 5 & 2.5634 & 6.5742 & 8 & 2.1798 & 386 \\
\hline & 7 & 5.0094 & 7 & 2.0822 & 6.0164 & 10 & 2.3101 & 4.2523 \\
\hline \multirow[t]{3}{*}{7} & 5 & 6.2768 & 2 & 3.8689 & 8.2804 & 6 & 2.5598 & 4.6606 \\
\hline & 6 & 6.0524 & 5 & 3.2871 & & 8 & 2.7953 & 347 \\
\hline & 7 & 5.8519 & 7 & 2.6702 & 7.0283 & 10 & 2.9624 & 4.9675 \\
\hline \multirow[t]{3}{*}{8} & 5 & 6.9840 & 2 & 4.7229 & 9.2133 & 6 & 3.1248 & 5.1857 \\
\hline & 6 & 6.7343 & 5 & 4.0127 & 8.5453 & 8 & 3.4123 & 5.3794 \\
\hline & 7 & 6.5112 & 7 & 3.2595 & 7.8202 & 10 & 3.6162 & 5.5271 \\
\hline \multirow[t]{3}{*}{9} & 5 & 7.5392 & 2 & 5.5780 & 9.9458 & 6 & 3.6906 & 5.5980 \\
\hline & 6 & 7.2697 & 5 & 4.7392 & 9.2247 & 8 & 4.0301 & 5.8071 \\
\hline & 7 & 7.0289 & 7 & 3.8496 & 8.4420 & 10 & 4.2710 & 5.9666 \\
\hline
\end{tabular}




\begin{tabular}{|l|l|l|l|l|l|l|l|l|}
\hline \multirow{3}{*}{10} & 5 & 7.9771 & 2 & 6.4337 & 10.5234 & 6 & 4.2567 & 5.9230 \\
\cline { 2 - 8 } & 6 & 7.6919 & 5 & 5.4662 & 9.7604 & 8 & 4.6483 & 6.1443 \\
\cline { 2 - 8 } & 7 & 7.4371 & 7 & 4.4402 & 8.9322 & 10 & 4.9262 & 6.3131 \\
\hline
\end{tabular}

Table 7: Empirical power of $A^{*}\left(k_{1}, k_{2}\right)$ for different values of $m, n, m_{1}^{+}, m_{2}^{+}, k_{1}, k_{2}$ and various distributions for $10 \%$ level of significance.

\begin{tabular}{|c|c|c|c|c|c|c|c|c|c|c|c|c|c|}
\hline \multirow[t]{2}{*}{$m_{1}$} & \multirow[t]{2}{*}{$m_{2}$} & \multirow[t]{2}{*}{$m_{1}^{+}$} & \multirow[t]{2}{*}{$m_{2}^{+}$} & \multirow[t]{2}{*}{$b$} & \multirow[t]{2}{*}{$d$} & \multirow{2}{*}{$\begin{array}{c}\text { Distribu } \\
\text { tion }\end{array}$} & \multicolumn{7}{|c|}{$\sigma$} \\
\hline & & & & & & & 1.2 & 1.5 & 2 & 2.5 & 3 & 4 & 5 \\
\hline \multirow[t]{5}{*}{6} & \multirow[t]{5}{*}{6} & \multirow[t]{5}{*}{2} & \multirow[t]{5}{*}{2} & \multirow[t]{5}{*}{2} & \multirow[t]{5}{*}{2} & Uniform & 0.1113 & 0.1949 & 0.2508 & 0.2896 & 0.3099 & 0.3466 & 0.3742 \\
\hline & & & & & & Normal & 0.1497 & 0.1971 & 0.2565 & 0.2940 & 0.3317 & 0.3651 & 0.3863 \\
\hline & & & & & & Logistic & 0.1415 & 0.1945 & 0.2394 & 0.2824 & 0.2997 & 0.3439 & 0.3650 \\
\hline & & & & & & Laplace & 0.1303 & 0.1698 & 0.2084 & 0.2469 & 0.2643 & 0.3040 & 0.3344 \\
\hline & & & & & & Cauchy & 0.1236 & 0.1348 & 0.1610 & 0.1854 & 0.1946 & 0.2173 & 0.2447 \\
\hline \multirow[t]{5}{*}{8} & \multirow[t]{5}{*}{8} & \multirow[t]{5}{*}{3} & \multirow[t]{5}{*}{3} & \multirow[t]{5}{*}{3} & \multirow[t]{5}{*}{3} & Uniform & 0.1124 & 0.2005 & 0.2514 & 0.2930 & 0.3273 & 0.3519 & 0.3790 \\
\hline & & & & & & Normal & 0.1516 & 0.2012 & 0.2688 & 0.3282 & 0.3484 & 0.3909 & 0.4067 \\
\hline & & & & & & Logistic & 0.1511 & 0.1942 & 0.2563 & 0.2917 & 0.3305 & 0.3793 & 0.3871 \\
\hline & & & & & & Laplace & 0.1410 & 0.1794 & 0.2249 & 0.2595 & 0.2876 & 0.3328 & 0.3558 \\
\hline & & & & & & Cauchy & 0.1302 & 0.1384 & 0.1681 & 0.1888 & 0.2099 & 0.2347 & 0.2549 \\
\hline \multirow[t]{5}{*}{16} & \multirow[t]{5}{*}{16} & \multirow[t]{5}{*}{8} & \multirow[t]{5}{*}{8} & \multirow[t]{5}{*}{2} & \multirow[t]{5}{*}{2} & Uniform & 0.0958 & 0.1941 & 0.2469 & 0.2818 & 0.2997 & 0.3211 & 0.3214 \\
\hline & & & & & & Normal & 0.1281 & 0.1663 & 0.2055 & 0.2157 & 0.2189 & 0.2092 & 0.1959 \\
\hline & & & & & & Logistic & 0.1265 & 0.1524 & 0.1780 & 0.1958 & 0.1944 & 0.1964 & 0.1783 \\
\hline & & & & & & Laplace & 0.1165 & 0.1329 & 0.1569 & 0.1722 & 0.1747 & 0.1742 & 0.1678 \\
\hline & & & & & & Cauchy & 0.0973 & 0.0968 & 0.0947 & 0.0958 & 0.0934 & 0.0851 & 0.0777 \\
\hline 16 & 16 & 8 & 8 & 3 & 3 & Uniform & 0.1004 & 0.1933 & 0.2456 & 0.2711 & 0.2776 & 0.2971 & 0.2926 \\
\hline & & & & & & Normal & 0.1293 & 0.1628 & 0.1961 & 0.2148 & 0.2095 & 0.1928 & 0.1774 \\
\hline & & & & & & Logistic & 0.1226 & 0.1510 & 0.1751 & 0.1863 & 0.1889 & 0.1752 & 0.1618 \\
\hline & & & & & & Laplace & 0.1174 & 0.1331 & 0.1506 & 0.1684 & 0.1653 & 0.1644 & 0.1568 \\
\hline & & & & & & Cauchy & 0.1014 & 0.1022 & 0.0890 & 0.0925 & 0.0923 & 0.0847 & 0.0769 \\
\hline 16 & 16 & 8 & 8 & 4 & 4 & Uniform & 0.1073 & 0.1949 & 0.2252 & 0.2431 & 0.2514 & 0.2506 & 0.2498 \\
\hline & & & & & & Normal & 0.1254 & 0.1565 & 0.1806 & 0.1946 & 0.1909 & 0.1745 & 0.1701 \\
\hline & & & & & & Logistic & 0.1242 & 0.1422 & 0.1685 & 0.1758 & 0.1741 & 0.1656 & 0.1579 \\
\hline & & & & & & Laplace & 0.1198 & 0.1323 & 0.1512 & 0.1576 & 0.1613 & 0.1599 & 0.1524 \\
\hline & & & & & & Cauchy & 0.1061 & 0.0995 & 0.1014 & 0.0998 & 0.0945 & 0.1041 & 0.0910 \\
\hline 16 & 16 & 8 & 8 & 5 & 5 & Uniform & 0.0998 & 0.1770 & 0.2101 & 0.2151 & 0.2077 & 0.2051 & 0.2104 \\
\hline & & & & & & Normal & 0.1272 & 0.1512 & 0.1777 & 0.1716 & 0.1718 & 0.1589 & 0.1571 \\
\hline & & & & & & Logistic & 0.1221 & 0.1418 & 0.1595 & 0.1671 & 0.1609 & 0.1543 & 0.1448 \\
\hline & & & & & & Laplace & 0.1235 & 0.1296 & 0.1497 & 0.1507 & 0.1564 & 0.1516 & 0.1451 \\
\hline & & & & & & Cauchy & 0.1094 & 0.1104 & 0.1055 & 0.1022 & 0.1044 & 0.1011 & 0.1032 \\
\hline
\end{tabular}




\section{References}

[1] Bhat, S. V. Studies in Nonparametric Inference, An unpublished Ph.D. thesis submitted to the Karnatak University, Dharwad, 1995.

[2] Bhat, S. V., Shindhe, S. D. and Joshi, V. B. A Class of Outlier Resistant Tests for Two-sample Scale Problem, International Journal of Agricultural and Statistical Sciences. 14 (2), 2018, 455 463.

[3] Bhat, S. V. and Shindhe, S. D. Distribution-free Tests Based on Subsample Maxima or Minima for Two-sample Scale Problem, Bulletin of Mathematics and Statistics Research. 7 (3), 2019, 20-35.

[4] Deshpande, J. V., Gore, A. P. and Shanubhogue, A. Statistical Analysis of Nonnormal Data, New Age International Publishers Limited, Wiley Eastern Limited; 1995, 48.

[5] Deshpande, J. V. and Kusum, K. A Test for Nonparametric Two-sample Scale Problem, Australian Journal of Statistics. 32, 1984, 16-24.

[6] Duran, B. S. A Survey of Nonparametric Tests for Scale, Communications in Statistics Theory and Methods. 5(14), 1976, 1287-1312.

[7] Kusum, K. A New Distribution-free Test for Two-sample Scale Problem. Journal of Indian Statistical Association, 23, 1985, 97-107.

[8] Kochar C. S. and Gupta R. P. Some Competitors of the Mood Test for the Two-sample Scale Problem, Communications in Statistics Theory and Methods, 15(1), 1986, 231-239.

[9] Kossler, W. and Narinder Kumar, An Adaptive Test for the Two-sample Scale Problem Where the Common Quantile may be Different from the Median, Statistical Methodology, 29, 2016, 10-17.

[10] Lehmann, E. L. Consistency and Unbiasedness of Certain Nonparametric Tests, Annals of Mathematical Statistics, 22, 1951, 165-179.

[11] Mahajan, K. K., Gaur, A. and Arora, A. A Nonparametric Test for a Two Sample Scale Problem Based on Subsample Medians, Statistics and Probability Letters, 81, 2011, 983-988.

[12] Mehra K. L. and Rao K. S. A Class of Two-sample Nonparametric Tests for Scale Based on Subsample Extrema, In: 80th Indian Science Congress conference held at Goa University, Goa, India, 1992.

[13] Mood, A. M. On the Asymptotic Efficiency of Certain Nonparametric Two-sample Tests, Annals of Mathematical Statistics, 25, 1954, 514-533.

[14] Narinder Kumar and Goyal, M. A General Class of Nonparametric Tests for Comparing Scale Parameters, Communications in Statistics Theory and Methods, 47 (24), 2018, 5956-5972.

[15] Rohatgi, V. K. and Saleh, A. K. M. E. An Introduction to Probability and statistics, 2 Ed., John Wiley and Sons, Inc; 2001.

[16] Siegel, S. and Tukey, J. W. A Nonparametric Sum of Ranks Procedure for Relative Spread in Unpaired Samples, Journal of American Statistical Association, 55, 1960, 429-444.

[17] Shetty, I. D. and Bhat, S. V. A Class of Distribution-free Tests for the Two-sample Scale Problem. In: Proceedings of II International symposium on Optimization and Statistics held at Aligarh Muslim University, Aligarh, India, Nov 2-4, 1993, 91-96.

[18] Shetty, I. D. and Pandit, P. V. A Note on a New Class of Distribution-free Tests for the Two Sample Scale Problem Based on Subsample Medians, Communications in Statistics Theory and Methods, 33, 2004, 2271-2280.

[19] Sukhatme, B. V. On Certain Two-sample Nonparametric Tests for Variances, Annals of Mathematical Statistics, 28, 1957, 188-194.

[20] Sukhatme, B. V. A Two-sample Distribution-free Test for Comparing Variances, Biometrika, 45, 1958, 544-548.

\footnotetext{
*Corresponding author.

E-mail address: bhat_sharada@yahoo.com
} 